\title{
Respecto a la escala de Glasgow Blatchford
}

\section{Regarding the Glasgow Blatchford scale}

\author{
Ana G. Beraun Cruz, Dayanna A. Figueroa-Pecho y Pedro J. Chunga-Tume* \\ Escuela Profesional de Medicina Humana, Universidad Privada San Juan Bautista, Chorrillos, Lima, Perú
}

El trabajo realizado por Olivarec, et al. evaluó la capacidad de escala de Glasgow Blatchford (GBS) para identificar el riesgo de resangrado a 30 días después de la hemorragia digestiva alta, encontrando que esta es útil; sin embargo, se encuentra bibliografía que contradice este estudio'.

Chang, et al., contrariamente, demuestra la importancia de la GBS para predecir la mortalidad, necesidad de transfusión de sangre y la necesidad de intervención endoscópica, sin embargo, esta no es útil para predecir el riesgo de resangrado, esto debido a que en el estudio de Olivarec, et al. excluyen el parámetro de característica de presentación y comorbilidades contrario a este estudio².

Stanley, et al. encuentran que el score de Progetto Nazionale Emorragia Digestiva (PNED) es superior a GBS para el resangrado, por la utilidad dentro de su puntuación del resangrado y endoscopia, así mismo predijo el resangrado en siete días, sin embargo concluye que ningún sistema predice el riesgo de resangrado ${ }^{3}$.

Paralelamente, Chandnani, et al. concluyen que PNED es mejor para evaluar el resangrado en comparación con la GBS, pero al comparar el PNED modificado (no incluye el resangrado en su puntuación) y la GBS ambos tuvieron el mismo desempeño ${ }^{4}$.

Similarmente, Lu, et al., quienes comparan GBS y la escala de Rockall (RS), la cual también utiliza la endoscopia, al omitir este parámetro (RS preendoscópica) sigue siendo mejor para predecir el resangrado ${ }^{5}$.

Por ello debemos considerar el uso de otras escalas como las ya mencionadas para el riesgo de resangrado para poder prevenir esto y otorgar tratamiento oportuno.

\section{Financiamiento}

Los autores declaran no haber recibido financiamiento para este trabajo.

\section{Conflicto de intereses}

Los autores declaran no tener conflicto de intereses.

\section{Responsabilidades éticas}

Protección de personas y animales. Los autores declaran que para esta investigación no se han realizado experimentos en seres humanos ni en animales.

Confidencialidad de los datos. Los autores declaran que han seguido los protocolos de su centro de trabajo sobre la publicación de datos de pacientes.

Derecho a la privacidad y consentimiento informado. Los autores declaran que en este artículo no aparecen datos de pacientes.

\section{Bibliografía}

1. Olivarec-Bonilla M, Herrera-Arellano AMGA. Riesgo de resangrado en hemorragia digestiva alta según la escala de Glasgow-Blatchford: herramienta de triaje. Gac Med Mex. 2020;156:502-8.

2. Chang A, Ouejiaraphant C, Akarapatima K, Rattanasupa A, Prachayakul V. Prospective comparison of the AIMS65 score, Glasgow-Blatchford score, and Rockall score for predicting clinical outcomes in patients with variceal and nonvariceal upper gastrointestinal bleeding. Clin Endosc. 2021;54(2):211-21.

3. Stanley AJ, Laine L, Dalton HR, Ngu JH, Schultz M, Abazi R, et al. Comparison of risk scoring systems for patients presenting with upper gastrointestinal bleeding: International multicentre prospective study. BMJ. 2017:356:1-8.

4. Chandnani S, Rathi $P$, Sonthalia N, Udgirkar S, Jain S, Contractor $Q$, et al. Comparison of risk scores in upper gastrointestinal bleeding in western India: A prospective analysis. Indian J Gastroenterol. 2019:38(2):117-27.

5. Lu M, Sun G. Huang H, Zhang X, Xu Y, Chen S, et al. Comparison of the Glasgow-Blatchford and Rockall scores for prediction of nonvariceal upper gastrointestinal bleeding outcomes in Chinese patients. Med (United States). 2019;98(21):1-5.
Gac Med Mex. 2021;157:570

Disponible en PubMed

www.gacetamedicademexico.com

CC BY-NC-ND (http://creativecommons.org/licenses/by-nc-nd/4.0/). 